\title{
On the choice issue of functional tests in physical fitness of the undergraduates
}

\author{
Valentina Talantseva ${ }^{1 *}$, Andrei Orlov ${ }^{2}$, Nadezhda Altynova ${ }^{1}$, and Andrei Platunov ${ }^{3}$ \\ ${ }^{1}$ Chuvash State Agrarian University, Cheboksary Town, Russia \\ ${ }^{2}$ Chuvash State University named after I. N. Ulyanov, Cheboksary Town, Russia \\ ${ }^{3}$ National University of Uzbekistan named after Mirzo Ulugbek, Tashkent Town, Uzbekistan
}

\begin{abstract}
Purpose: The purpose of our research is to determine, on the basis of mathematical processing of the experimental results, the most informative functional test, which makes it possible with a greater degree of reliability to assess the state of the vestibular apparatus and the presence of deviations in the work of the nervous system of students of higher educational institutions, and thereby recommend it for use in the practice of physical education in higher educational institutions. Results: Data analysis of the ascertaining experiment for evaluation of the condition of the vestibular system and possible nervous system disorder of the undergraduates shows a significant difference in functional test indicators which makes it necessary to choose the most informative one. Considering the comparison of the test results according to the normal distribution law and further calculation of variation coefficient of the received data it was defined that Romberg test example assuming to hold the balance standing on one leg with eyes closed with hands upwards is the most informative test allowing to more exactly define the condition of the vestibular system and nervous system of the undergraduates. Conclusion: Practical application of functional tests in physical training of students and interpretation of received data regarding the actual biological age of students allows us to actualize their need in physical activity increase. Herewith it is necessary to rely on results of the most informative functional tests, which do not require the application of complex measuring equipment and are quite simple in calculation of results.
\end{abstract}

\section{Introduction}

The modern stage of the development of the undergraduates' physical fitness is marked by a paradoxicality showing, that most students admit the necessity of physical exercises to be healthy, but stay physically passive $[1,2,3]$. On this account the specialists pay greater attention to the issue of increase of students' physical activity [4].

The current situation in the student's physical training can be explained within the framework of psychological theory of activity (L. S. Vygotskiy, A. N. Leontev, S. L. Rubinstein, Yu. B. Gippenreiter and other) according to the mechanism of

* Corresponding author: sport chieim21@mail.ru 
objectification of need. Considering the students' real age, it is obvious that the overwhelming majority of them do not feel considerably uncomfortable in their physical condition, and it doesn't motivate them to do physical exercises. At the same time according to the dialectic materialism the world around us (material environment, biological environment, social environment) is in constant motion. Whilst the process of changing is monodirectional in the material world and is of entropy nature, in the biological and social environments the choice of direction of the developmental process (progress and regress) shall be defined by the subject's action or inaction. In other words, in the context of the study even if to take no regular exercises at a young age the regressive processes begin to prevail in the human ontogenesis resulting in gradual decline in fitness shape.

For the purposes of the foregoing detection of means of needs objectification in intensifying the undergraduates' physical activity is topical. In this regard, the results of previous studies are of significant interest [5], whereby the correlation of indicators of functional tests with the biological age of the test people is a significant reserve in objectification of desired needs. It is noteworthy that graded credit is not applied in the academic curriculum in physical training and sport, which doesn't motivate the majority of students to increase their results in reaching qualifying standards. Therefore, this doesn't contribute to increase the students' motivation in physical training and sport, and improvement ofphysical fitness.

According to a range of scientists $[6,7]$ it is necessary to pay attention that functional tests when choosing them for application in students' physical training are informative, useable, require no complex measuring equipment.

If during determining the functional condition of the cardiovascular system the choice of tests which are in compliance with the present requirements causes no difficulties (Ruffier, Matrine-Kushelevskiy tests and other) $[8,9]$ when evaluating the condition of the nervous and vestibular systems the tests and ways of their implementation recommended in the scientific and methodological literature slightly differ therewith causing some difficulties in their application in the undergraduates' physical training.

\section{Purpose of study}

Considering the foregoing the purpose of the study is set forth as follows - to choose the most applicable functional test to evaluate the condition of the vestibular system and capacity of the nervous system of the undergraduates for application in physical training.

\section{Materials and methods}

According to the chosen topic of the study the analysis and integration of data of scientific and methodological literature, ascertaining experiment, mathematical processing of experimental data, and interpretation of the experiment results were the main methods.

Participants. 90 female students of the $1^{\text {st }}$ and $2^{\text {nd }}$ years of study of economic, veterinary as well as biotechnological and agronomic faculties of Chuvash State Agrarian University. The average age of the test people is 18.69 . According to their health condition $73.33 \%$ of the respondents were included to the general medical group, $16.67 \%$ to the special medical group, and $3.33 \%$ to the therapeutic group.

Organization of study. The study was conducted from September to October 2019-2020 academic year at the FSBEI HE Chuvash State Agrarian University. The evaluation of the condition of the vestibular system of the test people was made at the end of the introductory part of the physical training class. 
Usually, Yarotskiy test, Bondarevskiy test, and variation of Romberg test standing on one leg are applied in the evaluation of the condition of the vestibular system and nervous system disorder $[10,11]$. Yarotskiy test presupposes to keep the balance in normal standing position when exercising head circle movements in one direction 2 circles per second. The longer the test person keeps the balance the higher vestibular tolerance is. Under normal condition the test indicators make up 28 seconds. Bondarevskiy test is made in the standing position on one leg, arms at hips, with eyes closed. Another leg is bent and the heel is pressed to the inner surface of the knee joint of the standing leg. The principle of the Romberg test is to keep the balance standing on one leg similar to the Bondarevskiy test, but with arms upwards and fingers stretched at the most. In both tests the time of being in steady position is recorded which is defined including time without hand tremor. Under normal condition the test indicators make up 15 seconds $[8,12]$.

\section{Study results}

Results of the conducted study on evaluation of the condition of the students' vestibular system are shown in Table 1.

Table 1. Correlation of indicators of functional tests of female students of the Chuvash Agrarian University, $(n=90)$.

\begin{tabular}{|l|c|}
\hline \multicolumn{1}{|c|}{ Functional tests } & \multicolumn{1}{|c|}{$\boldsymbol{M} \pm \boldsymbol{m}^{*}$} \\
\hline Yarotskiy test, sec & $40.27 \pm 7.51$ \\
\hline Bondarevskiy test, sec & $10.10 \pm 2.00$ \\
\hline Romberg test, sec & $5.84 \pm 0.57$ \\
\hline $\begin{array}{l}* \text { Note: M - the arithmetic mean of the sample ; } \mathrm{m} \text { - standard error of the arithmetic mean (mean } \\
\text { error) }\end{array}$ \\
\hline
\end{tabular}

According to the results of the Yarotskiy test the test people have a well-developed vestibular system. The average value is almost by half above normal. However, the results of the Bondarevskiy and Romberg tests show the contrary. In the former case the average value is almost by half below normal, and the latter - almost three-fold. At the same time, according to the Student's t-test the test results of the Bondarevskiy and Romberg tests are significantly different $(\mathrm{P}>0.05)$ despite the fact that the test environment has no significant difference.

\section{Discussion}

Considering the received experiment results we have made an attempt to correlate them according to the normal distribution law, also known as the Gaussian curve.

$$
f(x)=\frac{1}{\delta \sqrt{2 \pi}} \exp \left(-\frac{(x-\mu)^{2}}{2 \delta^{2}}\right)
$$

As it can be seen from the data on figures 1-3 by the results of the Yarotskiy test assessment the dome of the Gaussian curve is too expanded horizontally (X-axis) (fig.1), and the standard deviation made up 41.12 as evidenced by the significant data difference. 


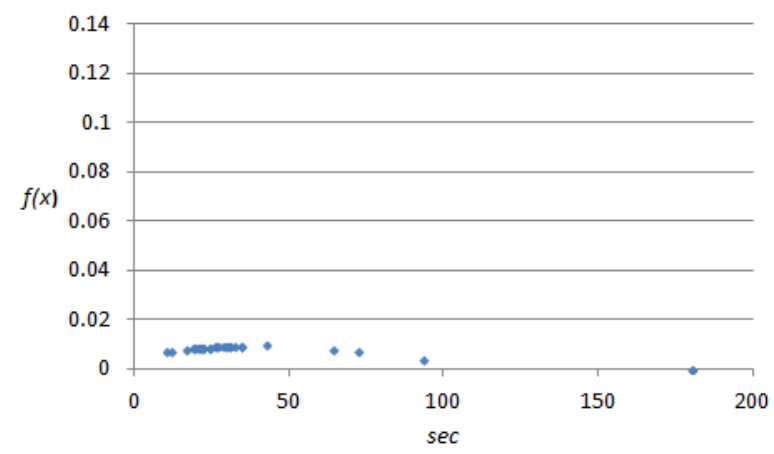

Fig. 1. Distribution of the Yarotskiy test indicators according to the Gaussian curve.

The Gaussian curve (fig.2) is more expressed in the Bondarevskiy test indicators, but similar to the Yarotskiy test indicators the detected standard deviation (10.94) evidenced a significant study data difference.

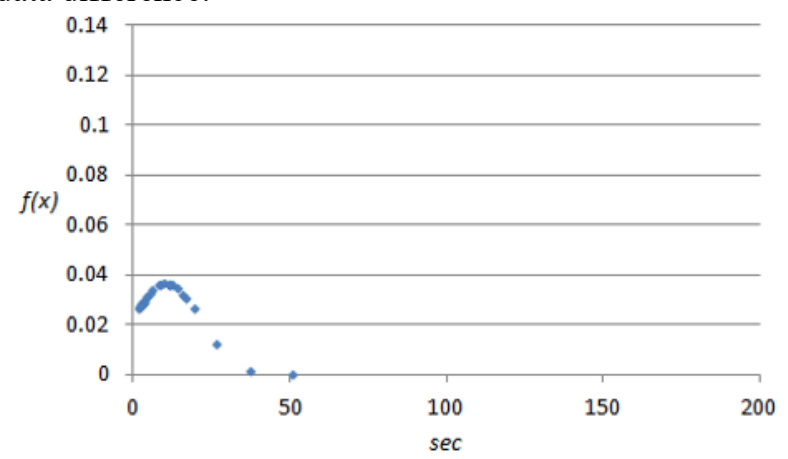

Fig. 2. Distribution of the Bondarevskiy test indicators according to the Gaussian curve.

The least standard deviation was detected in the Romberg test indicators (3.11), where the dome of the Gaussian curve is significantly extended vertically (Y-axis) (fig.3) as evidenced by the least indicators difference comparing to the results of two other functional tests. It is determined that the variation coefficient (correlation of standard deviation to average value expressed in per cent) of the Romberg test indicators are almost twice less comparing to the results of the Yarotskiy and Bondarevskiy tests.

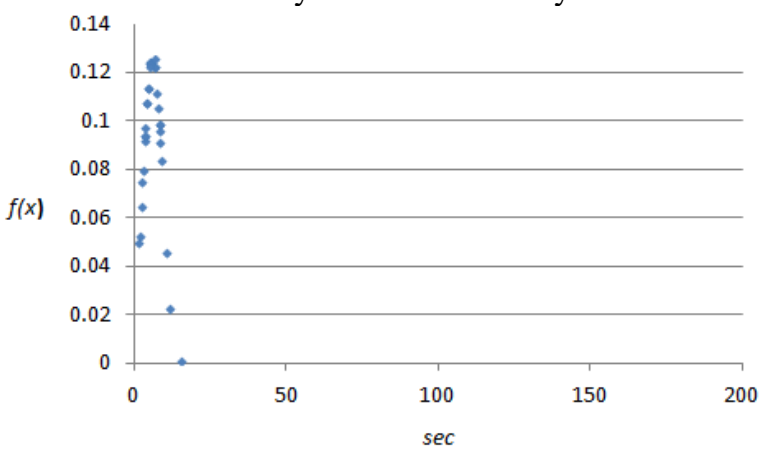

Fig. 3. Distribution of the Romberg test indicators according to the Gaussian curve. 
In our opinion, relatively bigger range of indicators difference of the Yarotskiy and Bondarevskiy functional tests is due to the fact that it is quite difficult for the test people to fulfil the conditions in the first test. The evidence from practice shows that range and tempo of head circle significantly differ among the test people. Such a test peculiarity makes it impossible to recommend the test for a wide practical application at the higher educational institution.

When keeping arms at hips during the Bondarevskiy test the centre of mass of the test people is slightly below than in the Romberg test. It results in some increase of stability corner and creates more favourable conditions in test execution and, consequently, does not allow to evaluate the nervous system and vestibular system dysfunctions more exactly. In other words, the Bondarevskiy test doesn't cause any difficulties for the undergraduates, and only relatively complex conditions of the Romberg test are more informative for the youth. It also should be noted that despite some difference in the received experimental data of the Bondarevskiy and Romberg tests and comparing them with biological age by Voytenko method $[13,14]$ no significant difference was detected. Indicators of the nervous and vestibular systems in the vast majority of test students correspond to those of adults. Thus, according to the Bondarevskiy test biological age of $56.67 \%$ of test people was 65 years, $20.00 \%$ - 60 years (figure 4 ), and according to the Romberg test results $66.67 \%$ of test people were over 65 years, and $23.33 \%-65$ years old (figure 5).

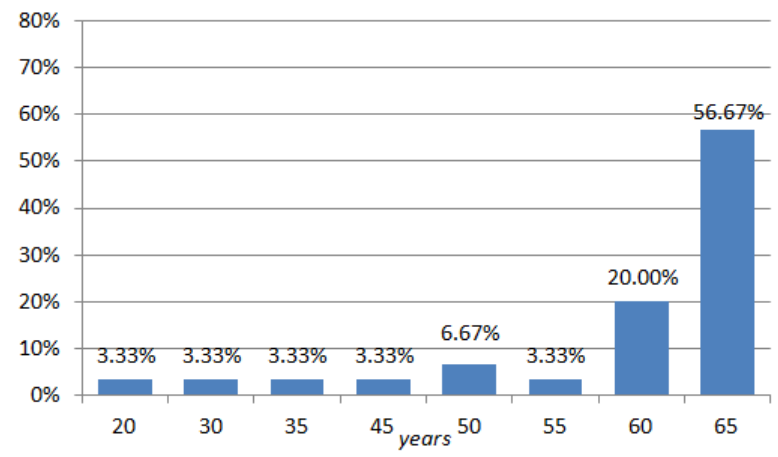

Fig. 4. Correlation of indicators of the Bondarevskiy test with indicators of biological age.

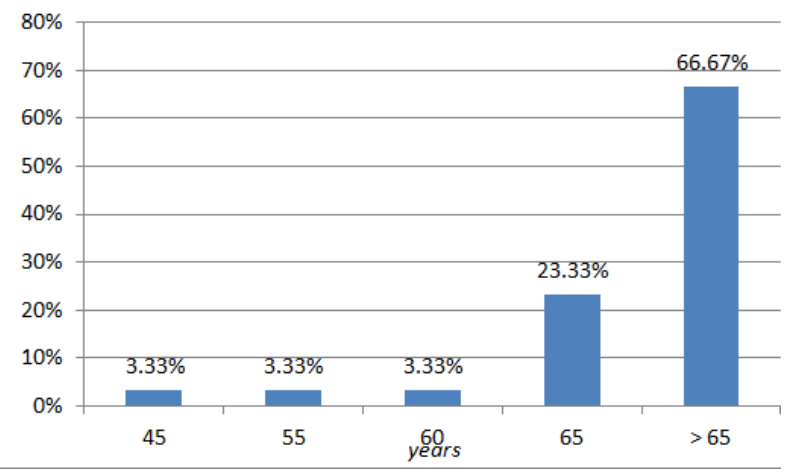

Fig. 5. Correlation of indicators of the Romberg test with indicators of biological age.

Results of the conducted experiment to some extend correlate with the study results of O. V. Parygina, Yu. A. Matveeva (2017) whereby balance function indicators of almost $70 \%$ of students were "unsatisfactory" [15]. It demonstrates both pronounced tendency towards worsening of vestibular system of modern undergraduates, and necessity to 
increase the amount of physical exercises aimed at developing balance and coordination abilities.

Analysis of the conducted experiment results by the example of evaluation of the condition of the vestibular system not only makes us think about undergraduates' fitness shape, but also to some extend contributes to fitness motivation since correlation of the received data with the indicators of biological age includes opportunity of the undergraduates' objectification of needs in physical exercises actualizing their actual physical fitness.

\section{Conclusion}

Analysis of scientific and methodological literature on the problem of increasing the physical activity of student youth, and personal long-term experience in teaching physical culture at a university, allows us to conclude that the traditional approach to organizing physical education of students does not fully meet modern requirements. All this can be explained within the framework of the position of the psychological theory actively objectifying the need, when subjective satisfaction in physical health does not induce the majority of students to physical activity. At the same time, the currently implemented elective disciplines (modules) in physical culture, which involve taking into account the individual preferences of students in one form or another of physical activity, do not provide a solution to the indicated problem.

In our opinion, the correlation of indicators of various functional tests with the biological age of students has a significant potential in increasing the physical activity of students. At the same time, for the convenience of their implementation in the practice of physical education, one should choose not those that would be distinguished by information content, ease of use and would not require complex measuring equipment. It should be noted here that while determining the state of a number of physiological systems of the body, the determination of functional tests does not cause particular difficulties, then when studying the vestibular apparatus and the nervous system of students, the opinions of researchers differ.

The results of the study allow us to state that, firstly, the variation of the Romberg test assuming to hold the balance standing on one leg is the most informative test among the variety of other tests allowing to define the condition of the vestibular and nervous systems of the undergraduates.

Secondly, currently determination of the students' biological age by the results of different functional tests can be an efficient means to involve them to regular physical exercises. This is difference between the received and expected test results which actualizes the students' need to increase their physical activity while indicators of physical activity can't do the same.

Thirdly, due to the steadily growing number of students who refer to the special medical and therapeutic groups the results of academic achievement in physical training and sport shall be taken into account both by indicators of physical fitness and positive dynamics of changes in functional tests indicators. This is due to the fact that the overwhelming majority of the student youth are not able to fully fulfil the requirements of physical training and sport curriculum for objective reasons. Therefore, instructors of the departments of physical training and sport have to assess students based on academic attendance and fulfilment of additional tasks (preparation and presentation of reports, participation in student scientific and practical conferences, and sporting and mass events, etc.). 


\section{Recommendation}

Analysis of scientific and methodological literature shows that interpretation of functional test results with regard to indicators of biological age shall be considered as a mechanism of undergraduates' objectification of needs in physical activity.

Due to a low level of fitness of the undergraduates it is considered reasonable to assess the academic results in physical training and sport both by indicators of physical fitness and dynamics of functional tests indicators for the whole period of study at the higher educational institution.

Results of the conducted study makes it possible to recommend application of the variation of the Romberg test standing on one leg in the physical training of students as the most informative for this age group, which requires no complex measuring equipment and prompt calculation of results.

Authors of the article herewith express their sincere gratitude to the administration of the Chuvash State Agrarian University for the study base for the experimental work.

\section{References}

1. V. K. Talantseva. Peculiarities of classes in Physical Training and Sport (elective discipline) for students of special medical group due to health condition: study guide / V. K. Talantseva, T. I. Volkova, N. V. Altynova. Cheboksary, 2018.

2. Osipov A, Kudryavtsev M, Fedorova P Serzhanova Z, Panov E, Zakharova L, Savchuk A, Yanova M, Zhavner T, Klimuk Y. Components of positive impact of exposure on university physical culture and sports on students' physical loads. Journal of Physical Education and Sport, 2017; 2: 871-878.

3. Samokish I, Bosenko A, Dishel' G. Optimization of teaching physical education at higher educational institutions on the basis of students' functional capacity monitoring. Naukaiosvita, 2016; 8: 151-157. - 3

4. Santana CCA, Azevedo LB Cattuzzo MT, Hill JO, Andrade LP, Prado WL. Physical fitness and academic performance in youth: A systematic review. Scandinavian Journal of Medicine \& Science in Sports. 2017; 27(6):579-603.

5. Altynova N.V. On improving the process of forming the physical readiness of future managers / N.V. Altynova, V.K. Talantseva // Proceedings of the First International Volga Region Conference on Economics, Humanities and Sports (FICEHS 2019). Advances in Economics, Business and Management Research, volume 114. - Paris : Atlantis Press, 2020. - P. 624-628.

6. Sergiienko L, Sharij D. Methodological bases of complex tests in physical education and sport. Teoriia ta metodikafizichnogovikhovannia, 2010; 5: 3-12.

7. Sutula V. Peculiarities of the use of statistical methods in scientifi c research in the fi eld of physical culture and sports. Teoriiaimetodikafizichnogovikhovanniaisportu, $2014 ; 1,82-86$.

8. O. Buykova. Functional tests in therapeutic and mass physical training: study guide / O. M. Buykova, G. I. Bulnaeva - Irkutsk: ISMU, 2017. - P. 24.

9. Sergiienko VM. The system of motor abilities control of student youth: the theory and methodology of physical education. Sumy: Sumy Condition University; 2015.

10. Iagotin R, Dehtiarenko T, Bosenko A. Complex Examination of University Students' Psychosomatic Health. Ukrainian Journal of Medicine biology and sports, 2017; 4(6): 223-228. 
11. Mantrova I. Methodical guide on psychophysiological and psychological diagnosis. Ivanovo: Neurosoft; 2007.

12. What is the Romberg test and it is able to detect: [on-line resource] URL: http://neurodoc.ru/diagnostika/simptomy/poza-romberga.html.

13. Do you know your Biological Age?: [on-line resource] URL: https://zen.yandex.ru/media/id/5ad254f20422b4c791a59b35/a-vy-znaete-svoibiologicheskii-vozrast-test-5b8ab5833c9a2400a9a120be.

14. A book of test to find your Biological Age: [on-line resource] URL: https://beautyaura.ru/tests/bioage/biological-age-test.html.

15. O. V. Parygina. Assessment of balance function in students of maritime educational institutions during academic process / O. V. Parygina, Yu. A. Matveev // Physical training. Sport. Tourism. Motional recreation. - 2017. - V. 2, No. 4. - P. 81-86. 